\title{
DRUG UTILIZATION EVALUATION OF ANTIDIABETIC DRUGS AMONG TYPE 2 DIABETES PATIENTS OF TAMIL NADU
}

\section{GEETHA P*, SHANMUGASUNDHARAM P}

Department of Pharmacy Practice, Faculty of Pharmacy, Vels University (VISTAS), Tamil Nadu, India. Email: Igeethapharma@gmail.com Received: 22 April 2017, Revised and Accepted: 26 May 2017

ABSTRACT

Objectives: The aim of the study was to assess the prescribing pattern of antidiabetic drugs in Type 2 diabetes outpatients visiting to Diabetes Centre, Chennai.

Methods: A prospective study was carried out by evaluating 115 prescriptions of antidiabetic drugs over the period of 4 months to assess the prescribing pattern of antidiabetic drugs and also drugs used for other complications of Type 2 DM.

Results: Totally, 115 patients were evaluated, 58 were of male and 57 were of female. An average number of drugs per encounter were found to be 4.47. An average number of antidiabetic drugs were found to be 2.56 . In this study, the most commonly prescribed oral hypoglycemic drug class as single-drug regimen was that of alpha-glucosidase inhibitors (16.326\%), dipeptidyl peptidase-4 (DPP-4) inhibitors (14.62\%), biquanides (12.9\%), thiazolidine diones (9.8\%), sulfonyl urea (7.82\%) and meglitinides (2.38\%), and in multi drug regimen metformin + alpha-glucosidase inhibitors $(11.56 \%)$ were commonly prescribed.

Conclusion: Most commonly used drug was alpha-glucosidase inhibitors, followed by DPP-4 inhibitors and biguanides. All the patients received combination therapy to achieve the glycemic control.

Keywords: Antidiabetic drugs, Oral hypoglycemic drug, Prescribing pattern

(C) 2017 The Authors. Published by Innovare Academic Sciences Pvt Ltd. This is an open access article under the CC BY license (http://creativecommons. org/licenses/by/4. 0/) DOI: http://dx.doi.org/10.22159/ajpcr.2017.v10i9.19342

\section{INTRODUCTION}

Diabetes mellitus (DM) is a chronic metabolic disorder characterized by disturbances in the metabolism of carbohydrate, fat, and protein due to varying degrees of insulin secretion either hyposecretion or insulin insensitivity, in which there are high blood sugar levels over a prolonged period that requires lifelong medical treatment and ongoing patient self-management and support to prevent acute complications and to reduce the risk of morbidity and mortality [1,2]. In India, 2000, the prevalence of diabetes was estimated to be 31.7 million, followed by China (20.8 million) and in the United States (17.7\%). It is predicted in India that the diabetes population may rise up to 79.4 million individuals by $2030[3,4]$. Prevention and treatment involves a healthy diet, physical exercise, avoid tobacco, and being a normal body weight. Drug utilization studies are important to optimize the drug use, and it serves as an important tool those are in need of receiving medication and cost-effective treatment. The main aim of the diabetes management study is to prevent the development of micovascular complications and reduction in patient financial cost.

\section{METHODS}

This was a prospective study carried out in outpatients of the Aruna Diabetes Centre, Chennai. Permission from Institutional Human Ethics Committee (Ref: IEC/PHD/2015/2016/01), Vels University, Chennai, was obtained to conduct the study at Aruna Diabetes Centre,Chennai. The sample size for this study was 115 patients with the duration of 4 months (July 2016 to October 2016). Type 2 DM patients of 18 years and above receiving antidiabetic drugs of new and existing cases were selected for participation after fulfilling the inclusion and exclusion criteria. We excluded Type 1 diabetes patients, pediatric and pregnant women, and patients not willing to participate in this study. After obtaining the informed consent, sociodemographic data along with the details of antidiabetic therapy, duration of treatment was recorded.
Type 2 diabetes patients receiving only one active ingredient defined as monotherapy, whereas patients receiving medication with more than one active ingredient were defined as combination therapy.

\section{RESULTS}

The prospective study involving 115 prescriptions of patients with Type 2 DM is visiting to Aruna Diabetes Centre, Chennai. Patients were divided into four groups on the basis of ages: Less than 40 years, $41-60$ years, above $61-80$ years, and above 80 years. The demographic characteristics of the patients were studied: Gender, age, duration of diabetes, and comorbidities. Out of 115 prescriptions analyzed, male were 58 (50.43\%), female were 57 (49.56\%), and the mean age of the sample was $54.73 \pm 12.43$ years (Table 1 ).

Out of 115 prescriptions, the total number of drugs prescribed to be 515 . In which, $57.09 \%$ were antidiabetic drugs, whereas $42.91 \%$ prescribed for diabetic complications and for hypertension, hypercoagulation, and peripheral neuropathy and thyroid disorders. During this study, Type 2 diabetes patients receiving a number of antidiabetic drugs vary from one of eight drugs. An average number of drugs received per patient were found to be 4.47 . The average number of antidiabetic drugs received per individual was found to be 2.56 . The study found a higher incidence of diabetes among adult patients, with a high incidence in the age group of $41-60$ years (58.56\% of the total) followed by the age group above $(28.7 \%$ of the total) (Table 2$)$. The average duration of diabetes was 11.44 \pm 7.11 (mean \pm standard deviation [SD]) (Table 3).

In this study, in single-drug regimen and multidrug regimen the most commonly prescribed oral hypoglycemic drug class was that of alphaglucosidase inhibitors (18.3\%) DPP-4 inhibitors (14.62\%), biquanides $(12.925 \%)$, thiazolidine danes $(9.8 \%)$, sulfonyl urea $(7.82 \%)$, meglitinides $(2.38 \%)$, sodium glucose transport, or inhibitors $(1.02 \%)$ 
were prescribed. In multi-drug regimen, biquanides + alpha-glucosidase inhibitors $(11.6 \%)$ biquanides + sulfonyl urea $(10.5 \%)$, biquanides + DPP-4 inhibitors (6.46\%), biquanides + meglitinides (1.7\%), sulfonyl urea+ alpha-glucosidase inhibitors $(0.68 \%)$, sulfonylurea+thiazolidine dione $(0.34 \%)$ were prescribed. In three drug combination, biquanides + sulfonyl urea + alpha-glucosidase inhibitors $(0.34 \%)$ and biguanides + thiazolidine diones+ alpha-glucosidase inhibitors $(0.34 \%)$ were prescribed. Insulin injection was prescribed to $2.52 \%$ of the total drug population (Tables 4 and 5).

Duration of diabetes plays an important role in patients suffering from a long time. A firm blood glucose control results in lesser incidence of complications such as nephropathy were related to the duration of diabetes. In this study, majority of patients fall under the category

Table 1: Gender categorization of the patients

\begin{tabular}{ll}
\hline Gender & Number of patients $\mathbf{n = 1 1 5}(\mathbf{\% )}$ \\
\hline Male & $58(50.43)$ \\
Female & $57(49.56)$ \\
\hline
\end{tabular}

Datas are expressed as number and percentage

Table 2: Demographic data of the patients

\begin{tabular}{ll}
\hline Age (years) & Number of patients $\mathbf{n = 1 1 5}(\mathbf{\%})$ \\
\hline$<40$ & $13(11.3)$ \\
$41-60$ & $67(58.26)$ \\
$61-80$ & $33(28.70)$ \\
$>80$ & $02(1.7)$ \\
\hline
\end{tabular}

Datas are expressed as number and percentage

Table 3: Distribution of the diabetic patients according to duration of years

\begin{tabular}{ll}
\hline Duration of years & Number of patients $\mathbf{n}=115$ (\%) \\
\hline New case & $5(4.3)$ \\
$1-5$ & $23(20)$ \\
$6-10$ & $29(25.17)$ \\
$11-15$ & $30(26.08)$ \\
$16-20$ & $15(13)$ \\
Above 20 & $13(11.3)$ \\
\hline
\end{tabular}

Datas are expressed as number and percentage

Table 4: Prescribing pattern of oral hypoglycemic drug as single drug regimen based on various classes of drugs

\begin{tabular}{lll}
\hline $\begin{array}{l}\text { Name of the } \\
\text { drugs }\end{array}$ & $\begin{array}{l}\text { Number of times } \\
\text { prescribed }\end{array}$ & $\begin{array}{l}\text { Percentage of total oral } \\
\text { hypoglycemic drug n=294 }\end{array}$ \\
\hline Metformin & 38 & 12.925 \\
Glimepiride & 15 & 5.1 \\
Glipizide & 1 & 0.34 \\
Glibenclamide & 2 & 0.68 \\
Gliclazide & 4 & 1.36 \\
Gliziphage & 1 & 0.34 \\
Voglibose & 48 & 16.326 \\
Acarbose & 5 & 1.7 \\
Miglitol & 1 & 0.34 \\
Pioglitazone & 29 & 9.8 \\
Vildagliptin & 28 & 9.52 \\
Linagliptin & 4 & 1.36 \\
Tenegliptin & 7 & 2.38 \\
Sitagliptin & 2 & 0.68 \\
Saxagliptin & 2 & 0.68 \\
Repaglinide & 7 & 2.38 \\
Canagliflozin & 2 & 0.68 \\
Dapagliflozin & 1 & 0.34 \\
\hline Datas are expressed & &
\end{tabular}

Datas are expressed as number and percentage of $11-15$ years $(26.08 \%), 6-10$ years $(25.17 \%)$ followed by $1-5$ years $(20 \%)$, and new case (4.3\%) (Table 3 ). In our study, minimum of 2 drugs prescribed to $6.07 \%$ patients, majority of prescriptions with 4 drugs $(27.83 \%)$ and 5 drugs $(25.22 \%)$ were prescribed and maximum of 8 drugs prescribed to $3.48 \%$ of prescriptions (Table 6). Cardio vascular complications were reported in $29.56 \%$ of patients and they were treated with various lipid-lowering drugs such as rosuvastatin $(2.92 \%$ of total drugs), atorvastatin (1.28\% of total drugs). Aspirin $(1.28 \%$ of total drugs), clopidogrel (1\%), and finofibrate (1 in number) were also prescribed to prevent heart-related problems. Out of 115 patients, 78 patients $(63.83 \%)$ had a family history of diabetes.

In this study, hypertension (42.6\%) was the common comorbidity observed. Of the total study population, $53 \%$ of patients were having coexisting illness, in which hypertension was the predominant. Hypertension was frequently associated with increased stiffness of large arteries, which often precedes macrovascular events.

Microvascular complications were detected in patients with neuropathy $15(13 \%)$, they were treated with alpha lipoic acid, benfotiamine, mecobalamin, folic acid, pyridoxine combination of drugs, gabapentin, pregabalin, and epalrestat. Nephropathy detected in 31 patients (26.95\%), they were treated with angiotensin II receptor blockers such as olmesartan (5 in numbers), losartan (5 in numbers), telmisartan (10 in numbers) were given to the patient and ( 3 numbers), ramipril (1 in number). Retinopathy detected in 19 patients (16.52\%) identified by fundoscopy 3 nethra.

In the present study, voglibose was the most commonly prescribed monotherapy, second commonly prescribed drug was metformin, pioglitazone and vildagliptin. Metformin was the only one antidiabetic drug prescribed commonly in both single-drug regimen and multidrug regimen.

\section{DISCUSSION}

In this drug utilization study, an attempt has been made to describe the current prescribing pattern of anti diabetic drugs of Type 2 diabetes patients at the general private diabetes center, Chennai, was prescribed almost equally to men (50.43\%) and women (49.56\%). The prevalence of Type 2 DM was maximum in the age group of 41-60 years (58.56\%). Similar studies were carried out in India in Indore city where the maximum patients are in the age group of 51-60 and 41-50 years [5]. The mean age of the patient was $52.84 \pm 1.0$. The average number of anti-diabetic drugs per prescription was 2.6. In our study, $67 \%$ of drugs prescribed as single-drug regimen and $33 \%$ as single drug regimen and multi-drug regimen. In other studies conducted in India were performed in five private clinics of a locality of Hyderabad, 74.5\% prescribed as monotherapy, and $24.5 \%$ as combination therapy [6].

The average duration of diabetes was $11.44 \pm 7.11$ (mean \pm SD). The duration of diabetes plays an important role in the management of diabetes. Patients who have $<5$ years could generally be managed with monotherapy while the patients having more than 5 years are required combination therapy. In our present study, only $24.3 \%$ of patients were present $<5$ years and $25.17 \%$ had the duration of more than $5-10$ years, $40.38 \%$ above 10 years, and the majority of the patients received 4 and 5 drugs in our study (Table 6). Another study carried out in India in the year 2015-16 by Haghighatpanah et al., patients $<5$ years, 5-10 years and $>10$ years of diabetes duration were $21.9 \%, 24.4 \%$, and $53.7 \%$, respectively. The above study indicates that the majority of the patients (79\%) had 1-2 antidiabetic drugs and $20.7 \%$ prescribed with 3-4 antidiabetic drug [7]. In our study, hypertension (42.6\%) was the common comorbidity observed. A similar study was conducted by Brian and Charles, hypertension $(42.2 \%)$ was the highest common comorbidity observed in Type $2 \mathrm{DM}$ in Nigeria, and the average number of drugs prescribed were $4 \pm 1.6$ [8].

In this present study, highly prescribed single-drug regimen was alphaglucosidase inhibitors $16.326 \%$ of total drug prescribed. Voglibose was predominantly prescribed in this category. Treatment with alpha- 
Table 5: Prescribing pattern of multi drug regimen of various classes of oral hypoglycemic drug

\begin{tabular}{ll}
\hline Name of the drugs & Number of times prescribed \\
\hline Metformin+sulfonylureas & $\begin{array}{l}\text { Percentage of total oral } \\
\text { hypoglycemic drug n=294 }\end{array}$ \\
Metformin+thiazolidine diones & 31 \\
Metformin+alpha-glucosidase inhibitors & 3 \\
Metformin+DPP-4 inhibitors & 34 \\
Metformin+meglitinides & 19 \\
Sulfonyl ureas+thiazolidine diones & 5 \\
Sulfonyl ureas+alpha-glucosidase inhibitors & 1 \\
Metformin+sulfonyl ureas+alpha-glucosidase inhibitors & 2 \\
Metformin+thiazolidine diones+alpha-glucosidase inhibitors & 1 \\
\hline
\end{tabular}

Data are expressed as number and percentage. DPP-4: Dipeptidyl peptidase-4

Table 6: Number of drugs prescribed in each prescription

\begin{tabular}{|c|c|c|c|c|c|}
\hline $\begin{array}{l}\text { Number of } \\
\text { drugs }\end{array}$ & $\begin{array}{l}\text { Number of } \\
\text { prescriptions }\end{array}$ & $\begin{array}{l}\text { Percentage of } \\
\text { prescriptions }\end{array}$ & $\begin{array}{l}\text { Number of prescriptions } \\
\text { containing oral hypoglycemic } \\
\text { drugs }\end{array}$ & $\begin{array}{l}\text { Number of prescriptions } \\
\text { containing insulin }\end{array}$ & $\begin{array}{l}\text { Number of prescriptions } \\
\text { containing other drugs with } \\
\text { oral hypoglycemic drugs }\end{array}$ \\
\hline 1 & - & - & - & - & - \\
\hline 2 & 7 & 6.07 & 8 & - & 6 \\
\hline 3 & 20 & 17.39 & 31 & 1 & 28 \\
\hline 4 & 32 & 27.83 & 72 & 4 & 52 \\
\hline 5 & 29 & 25.22 & 92 & 2 & 51 \\
\hline 7 & 11 & 9.57 & 38 & 4 & 35 \\
\hline 8 & 4 & 3.48 & 15 & 1 & 16 \\
\hline
\end{tabular}

glucosidase inhibitor delays carbohydrate absorption after meals, significantly reduced post-meal hyperglycemia, along with the risk of myocardial infarction and other cardiovascular events with Type 2 diabetes [9] and impaired glucose tolerance [10].

The study conducted by Talaviya et al. alpha-glucosidase inhibitors prescribed as an add-on therapy with metformin and sulfonylureas in uncontrolled obese and overweight patients of Type 2 DM provide desired glycemic control, improvement in lipid profile, and decreases in body weight [11]. Metformin + alpha-glucosidase inhibitors (11.56\%) prescribed highest in our study.

Next, to alpha-glucosidase inhibitors, $14.62 \%$ of patients received dipeptidyl peptidase-4 (DPP-4) inhibitors. The study documented higher number of DPP-4 inhibitors such as vildagliptin (9.52\%) prescribed. DPP-4 inhibitors use has greatly increased with uncontrolled overweight/obese; this result is in agreement with other reports conducted worldwide [12-14]. Another study carried out by Tolba et al., vildagliptin as an add-on therapy to gliclazide was not associated with the cardiovascular risks and showed the significant reduction in the hemoglobin A1c and fasting blood sugar [15].

In our study, metformin $12.925 \%$ were prescribed as a single drug, and as in combination therapy, $31.96 \%$ were prescribed, almost in all the prescription metformin was present. According to the statement of the American Diabetes Association and European Association for the Study of Diabetes guidelines also endorses metformin is the first-line drug for Type 2 diabetes patients and has the lowest risk of hypoglycemia, it does not cause weight gain and weight loss, has the beneficial effect of lowering the lipid levels and available at a low cost $[16,17]$. The study conducted by different groups reported that an increase in the use of metformin and decrease in the use of sulfonylureas [18-21]. The addon therapy of sulfonylurea to metformin is the common procedure after the metformin fails to control glycemic levels, sulfonylureas have been associated with hypoglycemia, sometimes need of hospitalizations, particularly in elderly patients [22].

\section{CONCLUSION}

Among all the anti-diabetic drugs, class of alpha-glucosidase inhibitors, DPP-4, and metformin combinations played an important role in the maintenance of glycemic levels in Type 2 diabetes patients. We could see the decrease in the use of sulfonyl ureas; it causes weight gain and increases in the risk of cardiovascular complications. All the prescriptions, receiving a single-drug regimen as well as multiple drug regimen. All the prescriptions were dispensed with minimum 2 and maximum of 8 drugs. The number of drugs prescribed to patients increased due to their complications and existing comorbidities. Further investigation is needed to study the patient compliance and educations regarding diabetes, and lifestyle modification is also important to achieve the optimal glycemic control.

\section{ACKNOWLEDGMENTS}

The authors would like to thank Aruna Diabetes Centre, Choolaimedu, Chennai.

\section{REFERENCES}

1. American Diabetes Association. Standards of medical care in diabetes. Diabetes Care 2013;36 Suppl 1:11-66.

2. WHO, Consultation. Definition, Diagnosis and Classification of Diabetes Mellitus and its Complications. Geneva, Switzerland: World Health Organization; 1999. p. 31-3.

3. Wild S, Roglic G, Green A, Sicree R, King H. Global prevalence of diabetes-estimates for the year 2000 and projections for 2030. Diabetes Care 2004;27(3):1047-53.

4. Whiting DR, Guariguata L, Weil C, Shaw J. IDF diabetes atlas: Global estimates of the prevalence of diabetes for 2011 and 2030. Diabetes Res Clin Pract 2011;94(3):311-21.

5. Vengurlekar S, Shukla P, Patidar P, Bafna R, Jain S. Prescribing pattern of antidiabetic drugs in Indore city hospital. Indian J Pharm Sci 2008;70(5):637-40.

6. Rani J, Reddy S. Prescribing pattern of antidiabetic drugs in urban population of Hyderabad. Natl J Physiol Pharm Pharmacol 2015;5(1):5-9.

7. Haghighatpanah M, Thunga G, Jha A, Mallayasamy S. Study on prescrining pattern of anti-diabetic drugs among the 2 diabetes patients with complications in South Indian teaching hospital. Asian J Pharm Clin Res 2016;9(1):194-7.

8. Brian OO, Charles CE. Drug use indicators in patients with Type 2 diabetes in a tertiary health care facility in Nigeria. Int $\mathrm{J}$ Pharm Sci 2014;6(11):493-5.

9. Hanefeld M, Cagatay M, Petrowitsch T, Neuser D, Petzinna D, Rupp M. Acarbose reduces the risk of myocardial infraction in Type 2 
diabetic patients:meta analysis of seven long-term studies. Eur Heart J 2004;25(1):10-6.

10. Chiasson JL, Josse RG, Hanefeld M, Laakso MK. Acarbose treatment and the risk of cardiovascular disease and hypertension in patients with impaired glucose tolerance: The STOP-NIDDM trial. J Am Med Assoc 2003;290(4):486-94

11. Talaviya PA, Saboo BD, Dodiya HG, Rao SK, Joshi SR, Modh VB, et al. Retrospective comparision of voglibose or acarbose as an add-on therapy to sulphonylureas in Western Indian patients with uncontrolled overweight/obese Type 2 diabetes. Diabetes Metab Syndr 2016;10(2):88-91

12. Oishi M, Yamazaki K, Okuguchi F, Sugimoto H, Kanatsuka A, Kashiwagi A, et al. Changes in oral antidiabetic prescriptions and improved glycemic control during the years 2002-2011 in Japan (JDDM32). J Diabetes Investig 2014;5(5):581-7.

13. Hampp C, Borders-Hemphill V, Money DG, Wysowski DK. Use of antidiabetic drugs in the U.S., 003-2012. Diabetes Care 2014;37(5):1367-74

14. Zaharan NL, Williams D, Bennett K. Prescibing of antidiabetic therapies in Ireland: 10 year trends 2003-2012. Ir J Med Sci 2014;183(2):311-8.

15. Marwa K, Tolba A, EL Khashab KA, Said AS. The effect of dipeptidyl peptidase -4 inhibitors on cardiovascular disease risk in Type 2 diabetes mellitus. Int J Pharm Sci 2017;9(1):254-9.

16. Inzucchi SE, Bergenstal RM, Buse JB. Management of hyperglycemia in Type 2 diabetes: A patient centered approach. Position statement of the American diabetes association (ADA) AND THE European association for the study of diabetes (EASD). Diabetologia 2012;55:1577-96.

17. American Diabetes Association. 7. Approches to glycemic treatment. Diabetes Care 2016;39 Suppl 1:S52-9.

18. Baviera M, Monesi L, Marzona I, Avanzini F, Monesi G, Nobili A, et al. Trends in drug prescriptions to diabetic patients from 2000 to 2008 in Italy's Lombardy region: A large population-based study. Diabetes Res Clin Pract 2011;93(1):123-30.

19. Chang $\mathrm{CH}$, Jiang YD, Chung $\mathrm{CH}$, Ho LT, Chuang LM. National trends in anti-diabetic treatment in Taiwan, 2000-2009. J Formos Med Assoc 2012;111(11):617-24.

20. Leal I, Romio SA, Schuemie M, Oteri A, Sturkenboom M, Trifirò G. Prescribing pattern of glucose lowering drugs in the United Kingdom in the last decade: A focus on the effects of safety warnings about rosiglitazone. Br J Clin Pharmacol 2013;75(3):861-8.

21. Turner LW, Nartey D, Stafford RS, Singh S, Alexander GC. Ambulatory treatment of Type 2 diabetes in the U.S., 1997-2012. Diabetes Care 2014;37(4):985-92.

22. Lipska KJ, Ross JS, Wang Y, Inzucchi SE, Minges K, Karter AJ, et al. National trents in US hospital admissions for hypoglycemia and hypoglycemia among Medicare beneficiaris, 1999 to 2011. JAMA Intern Med 2014;174(7):1116-24 